\title{
Efficacité et protection des données grâce au «secure e-mail» également pour les caisses-maladie
}

\author{
Pour les assureurs-maladie et -accident, la protection des données lors de \\ communications électroniques est une préoccupation centrale. Swica - un des \\ plus importants assureurs-maladie et -accident de Suisse avec plus d'un million \\ d'assurés - a trouvé une solution sécurisée pour ses e-mails grâce au Mail \\ Gateway HIN.
}

Andreas Nievergelt

Correspondance: Andreas Nievergelt Health Info Net AG Pflanzschulstrasse 3 CH-8400 Winterthur Tél. 0522351934

andreas.nievergelt@hin.ch

\section{Problématique}

L'utilisation de messages électroniques dans le domaine de la santé est limitée. La loi exige une protection élevée sur les données sensibles liées aux patients. De nombreux échanges de données sont effectués entre les assurances et les hôpitaux ou les cabinets médicaux. Pour éviter de renoncer aux avantages de la messagerie électronique, Swica a décidé de se doter des bons outils. Dans trois départements de l'assurance des plans concrets sont établis afin de mettre en place une solution qui permette une utilisation quotidienne du «secure e-mail»:

1. modèles Managed Care: les médecins généralistes font ici office de centralisateur «Gatekeeper». Ils reçoivent ainsi mensuellement de l'assurance des informations sur l'état administratif actuel et les conditions des prestations de leurs propres patients selon le modèle Managed Care. Ces informations étaient expédiées auparavant par poste avec des coûts considérables;

2. centre de santé: les sept centres de santé communiquent régulièrement avec les hôpitaux et des experts externes. Les documents sont toujours envoyés par poste;

3. Care Management: le domaine Care Management est également régulièrement en contact avec les hôpitaux et les cabinets médicaux. Les collaborateurs et collaboratrices requièrent des lettres de sortie et d'autres documents médicaux sensibles. Care Management reçoit, en outre, toutes les demandes de garantie.

Ces trois départements spécialisés voulaient transférer, dès que possible, une partie de leurs communications vers une messagerie électronique sécurisée et conforme à la protection des données.

\section{Solution}

Le département technique de Swica s'est donc renseigné sur les différentes possibilités de mettre en place un service e-mail sécurisé. Il leur a fallu peu de temps pour choisir le «secure e-mail» de HIN. Quatre éléments ont contribué à ce choix :

- 80 pour cent des médecins en pratique privée ont déjà souscrit un abonnement HIN;

- 115 hôpitaux et autres organisations du domaine de la santé disposent également d'un «secure e-mail» $\mathrm{HIN}$;

- tous les collaborateurs de l'assurance peuvent être intégrés dans le domaine de messagerie sécurisée, qui est conforme à la loi sur la protection des données;

- le MailGateway (MGW) crypte également les e-mails échangés en interne entre les différents départements et entre les différents centres de santé Swica.

Le MGW a été installé rapidement et sans conséquences négatives dans le système informatique existant. Il gère le cryptage ou décryptage de tous les messages sécurisés.

\section{Conclusion}

Swica peut maintenant communiquer électroniquement, de manière conforme à la protection des données, depuis plus de 40 endroits. Tous les messages qui sont envoyés aux domaines sécurisés «@hin.ch»ou à d'autres domaines MGW (p.ex.«@sec.insel.ch») sont automatiquement 
cryptés. Ceci est également valable pour tous les e-mails envoyés avec le nom de domaine «sec.swica.ch».

Les trois départements spécialisés de Swica ont déjà adapté leur mode quotidien de travail:

- le département d'assurance alternative est aujourd'hui en mesure d'envoyer électroniquement, en deux clics de souris, les différentes mises à jour mensuelles, notamment celles liées aux prestations;

- les sept centres de santé peuvent effectuer leurs échanges de données avec des spécialistes externes et les hôpitaux via e-mail sécurisé;

- le département Care Management reçoit de plus en plus de lettres de sortie et autres documents sensibles par e-mail sécurisé. Il en va de même pour les demandes de garantie.

\section{Développement de I'utilisation}

Les réactions, suite à l'installation d'une solution «secure e-mail», sont positives. La communication électronique prendra vraisemblablement encore plus d'importance au cours des prochaines années. D’une manière générale, un changement culturel vers la communication électronique s'effectue dans les hôpitaux et les cabinets médicaux. D'autres modèles de travail internes aux assurances pourront être optimisés grâce à l'utilisation du «secure e-mail».

\section{MailGateway HIN}

Le MailGateway (MGW) HIN est aux hôpitaux ce que l'abonnement HIN est aux médecins. Un MailGateway fait office de serveur de messagerie spécialisé directement chez le client. Si un utilisateur interne désire envoyer un e-mail sécurisé à un abonné HIN, le mail est automatiquement transféré par le serveur de messagerie existant (p.ex. MS Exchange) vers le MailGateway. Celui-ci récupère la clé publique du destinataire au centre de calcul HIN (Public Key Infrastructure) afin de crypter le mail. Le MailGateway utilise un procédé de cryptage puissant d'au moins 1024 bit (standard S/MIME). Le message crypté est ensuite acheminé par internet et ne peut être décrypté que par le destinataire choisi. 